\title{
Effectiveness of Power Harvesting in Hierarchical Routing Protocols in Wireless Sensor Networks
}

\author{
M. A. Mohamed \\ Assoc. Prof. - Faculty of \\ Engineering \\ Mansoura University \\ Mansoura, 35511 - Egypt
}

\author{
M. M. Abdel-Razzak \\ Professor- Faculty of \\ Engineering \\ Mansoura University \\ Mansoura, 35511 - Egypt
}

\author{
A. K. Tawfeec \\ M.Sc. Student- Faculty of \\ Engineering \\ Mansoura University \\ Mansoura, 35511 - Egypt
}

\begin{abstract}
Wireless sensor networks (WSNs)consist of a large number of power constrained inexpensive sensor nodes, which aggregate data from the environment and transmit to sink node or base station, saving energy; lifetime; energy efficiency; fault tolerance; deployment of nodes, and latency have become the main great challenges in WSNs due to its wide range of applications. Clustering in routing protocols is a key technique optimize energy consumption in WSNs. In this paper, we propose routing protocol prolong stability and life time of the network. Hence, with the increased complexity of the application, WSN heterogeneity and energy level are also increased. Simulation of 100 sensor node in a field of $100 \times 100 \mathrm{~m}$, nodes are deployed random, base station is centered in the field and have unlimited power, hence ignoring the effect caused by interference and signal collision in the wireless channel was carried out. Simulation results show that the proposed routing protocol performs better performance measures than all presented traditional techniques: LEACH, SEP, TEEN, DEEC and DDEEC with more stability and effective messages.
\end{abstract}

\section{Keywords}

Wireless Sensor Networks (WSNs), Quality of Service (QoS), Low Energy Adaptive Clustering Hierarchy (LEACH), Stable Election Protocol (SEP), Energy Efficient Sensor Network (TEEN), Distributed Energy Efficient Clustering (DEEC).

\section{INTRODUCTION}

Wireless sensor network (WSN) has reached in recent years the most fields like [1, 2]: environment monitoring (habitat monitoring and hazard monitoring), military applications (battle field monitoring, object protection and remote sensing), home intelligence (smart home and remote metering), industrial process control and medical monitoring. WSN consist of autonomous battery powered sensor nodes, which can detect or monitor physical parameters, e.g. light; sound; pressure; temperature, etc. Sensor nodes are limited on sense; transmit; store; compute; board processing, and receive data from the environment. This sensors observe the physical phenomenon and generate analog signals based on the observed phenomenon. Sensor nodes sense and transmit their information or aggregate data to the sink or base station (BS) placed on center of the grid for assessment. Sensor Node structure[3-5]; (i) Power unit; battery; (ii) Sensing unit; sensor and analog-to-digital converter (ADC); (iii) Process unit; memory and microprocessor/microcontroller, and (iv) Communication unit; Radio, as shown in Fig.1.

The main problem in routing in WSNs are the battery; storage; capability of computing; data processing, and storage of sensor node are limited. How to reduce energy consumption and how to prolonging the network lifetime are the key problem. That is the main reason to perform the network with clustering algorithms $[6,7]$, these algorithms proposed for the energy of sensor nodes dissipated when base station receive redundant information from this node. Designers must carefully consider which are of particular importance in wireless sensor networks [8-10]: (i) Cost of Clustering, (ii) Election of Clusters and Cluster heads, (iii) Real-Time Operation; (iv) Cluster management (Synchronization); (v) Data gathering; (vi) Repair method, and (vii) Quality of Service (QoS) [11].Clustering parameter [10]: (i) Nodes and cluster head (CH) mobility: Various published approaches assumed the sensor nodes to be stationary, such networks are stable thus it is easy to maintain intercluster and intracluster Communication [8]. But in case of sensor node mobility we need to reelect the $\mathrm{CH}$ periodically and maintain cluster organization continually; (ii) Type of nodes: sensor nodes are of two types based on clustering approaches. In Homogenous network all sensor nodes have same functionality and in Heterogeneous network some sensor node are equipped with higher capabilities and complex hardware; (iii) Cluster count: cluster count can be fix or variable depending up on which clustering technique is used [12]. In probabilistic and randomized approaches $\mathrm{CH}$ are not predetermined thus the cluster formation process result in to variable no. of clusters. Cluster count is fixed for approaches where the $\mathrm{CH}$ is predetermined; (iv) Cluster-head election: Various published approaches adopt various criteria for selection of $\mathrm{CH}$. The sensor node in every cluster elects a leader among all the node either on randomized basis or follow a probabilistic approach or based on some other criteria (such as based on residual energy, node degree etc.); (v) Cluster formation process: cluster formation technique are of two type centralized or distributed earlier approaches followed centralized or hybrid approach, when $\mathrm{CHs}$ are just one or more coordinator nodes are used to partition the whole network off-line and control the cluster membership [13]. But nowadays as time efficiency is important distributed approach is followed; (vi) Communication among nodes: In clustering two type of communication can occur intercluster communication or intracluster communication both can be further of two type single hop and multi hop. Earlier clustering approaches assume the communication among its nodes and $\mathrm{CH}$ to be single hop but nowadays various approaches are published which provide multihop communication in intracluster, and (vii) Overlapping: Overlapping in clustering is said to occur when a sensor node is shared by more than one cluster. Overlapping provide better routing efficiency and also fasten up cluster formation process [14]. Some published approaches allow overlapping, some try to have minimum overlap some not at all permit overlaps.

Routing in wireless sensor networks differs from conventional routing in fixed networks in various ways. There is no 
infrastructure, wireless links are unreliable, sensor nodes may fail, and routing protocols have to meet strict energy saving requirements [15]. Many routing algorithms were developed for wireless networks in general. All major routing protocols proposed for WSNs may be divided into this categories [1622] as shown in Fig.2. Hierarchal routing is technique knows very well with special advantages relative to scalability and communication efficiently. The main issues in WSNs [5]: (i) Energy Efficient: This is the most important factor for any issue in the sensor networks; (ii) Scalability: a good MAC protocol easily entertains changes in density, topology and network size. Some nodes may die over time and new nodes may join later and some nodes may move to other locations; (iii) Fairness: in traditional wireless data or voice networks, each user wants time to access the medium and equal opportunity means, receiving and sending packets for their own applications. However, in sensor networks, all nodes cooperate for a single task and normally there is only one application running at any time. In this case fairness is not important as long as application-level performance is not degraded, and (IV) Latency: latency can be significant or insignificant it depends on what application is running and the state of node. At the time when there is no sensing event, normally there is very little data flowing in the network and nodes are in idle state most of the time. We can trade off for energy savings by allow the node to turn off their radios to decrease the energy consumption due to idle listening. The design of routing protocols for WSNs is challenging because of several network constraints. WSNs suffer from the limitations of several network resources, for example, energy, bandwidth, central processing unit, and storage $[33,34]$. The design challenges in sensor networks involve the following main aspects [22-26]: (i) scalability; (ii) Node Deployment; (iii) Node/Link Heterogeneity; (iv) Data Reporting Method; (v) Node Capabilities; (vi) Coverage Area; (vii) Power Consumption; (viii) Data Aggregation; (ix) Connectivity; (x) Fault Tolerance; (xi) Node Dynamics, and (xii) Transmission Media.

There are two types of network clustering schemes. Firstly, clustering algorithms applied on homogeneous networks, where all nodes have same initial energy like Low Energy Adaptive Clustering Hierarchy (LEACH) [27-30] used the probabilistic method to elect the $\mathrm{CH}$ and the probability of choosing the cluster head decides after how many rounds a node can be again cluster head. Secondly, clustering algorithms applied on heterogeneous networks, where a few sensor nodes have high initial energy from others like stable election protocol (SEP) [5]. Which network divided into two types of nodes having different initial energy level normal and advanced nodes? The probabilistic method of elect the cluster heads is based on their energies. This improves the stability period and prolongs the lifetime of the network

Various enhanced versions of SEP routing protocols were proposed like ESEP [28], ZSEP [31] etc. ESEP used three heterogeneity levels using the normal, intermediate and advanced nodes where increasing initial energies respectively. ZSEP divided WSN network form to zones which resemble clusters so that proper distribution of the nodes and energies in each cluster can be done These make ZSEP better than the SEP but weakness of these protocols including SEP of not set eyes on the effect of residual energies on the $(\mathrm{CH})$ selection probabilities for different type of nodes. Distributed energy efficient clustering (DEEC) protocol applied concept of considering the effect of residual energy and average energy of the network to elect $\mathrm{CHs}$ with existing heterogeneity in the network sensor nodes. This improve in stability period and prolong the lifetime of the network. DDEEC is developed routing algorithm from DEEC uses same approach for estimate average energy of the wireless sensor network and $\mathrm{CH}$ election algorithm based on residual energy. DDEEC insert threshold residual energy and took the concept of when energy level of two types of nodes (advanced and normal) reach to the limit of threshold residual energy in this case advance nodes and normal nodes have the same probability to elect as cluster head. Threshold Sensitive Energy Efficient Sensor Network (TEEN) [5, 27] protocol which classified as reactive protocol proposed method to reduce the transmission time as the processing of data in sensor nodes consume energy less than transmission. In this scheme, every round the cluster head broadcasts to its member's hard and soft threshold. A node transmits only when sensed value is more than hard threshold and difference between sensed current value and previous sensed value is more than soft threshold.

The remaining of this paper is organized as: (2) discussion of traditional energy harvesting algorithms; (3) presents the proposed algorithms; (4) introduces the results and discussions, and (5) provides the main conclusions and future works.

\section{TRADITIONAL WSNS ALGORITHMS}

\subsection{TEEN and LEACH Protocols}

LEACH and TEEN follow self-organizing and adaptive $\mathrm{CH}$ selection criteria. In setup phase, $\mathrm{CH}$ is elected on the bases of following threshold equation1 [32]; Fig.3 and Fig.4.

$$
T(n)=\left\{\begin{array}{cc}
\frac{p}{1-p\left(\operatorname{rmod} \frac{1}{p}\right)} & \text { ifn } \in G \\
0 & \text { otherwise }
\end{array}\right.
$$

Where $\mathrm{p}$ is the desired number of $\mathrm{CHs}, \mathrm{r}$ is the current round and $\mathrm{G}$ is the set of nodes that have not been $\mathrm{CH}$ in the current epoch. Epoch is the number of rounds for a $\mathrm{CH}$, after which again it become eligible to become a $\mathrm{CH}$. Each node generates a random number between 0 and 1 , if the number is less than the node's threshold, then this sensor node becomes a $\mathrm{CH}$. After the election of $\mathrm{CHs}$, each $\mathrm{CH}$ advertises its status using CSMA MAC protocol. Node selects its $\mathrm{CH}$, on the bases of RSSI and link quality of all $\mathrm{CHs}$, existing in range of that node. All nodes send their membership willingness message to the suitable $\mathrm{CH}$, using CSMA MAC. Then $\mathrm{CHs}$ schedule all nodes using TDMA for data transmission. In steady-state phase, each node transmits its data to their respective $\mathrm{CH}$ in specific allocated time slots. $\mathrm{CH}$ then aggregates data and sends the compressed data to BS.

\subsection{SEP Protocol}

SEP is a protocol for heterogeneous network; heterogeneity in terms of initial energy deployment in WSNs. SEP assumes that in real environment nodes have different energy, therefore SEP two types of nodes, advance nodes and normal nodes. Advance nodes have an amount of more energy than normal nodes. SEP assign a weighted probability to each node based on its initial energy. Moreover, it improves the cluster formation of LEACH by decreasing the $\mathrm{CH}$ epoch interval of advance nodes, advance nodes get more chances to become a $\mathrm{CH}$. LEACH threshold formula in Eq.2 works well for homogeneous energy nodes, however, the problem of maintaining well distributed energy consumption constraints, in the stable period arises in heterogeneous energy nodes environment. SEP resolve this issue by introducing guaranteed well distributed energy consumption constraint in the stable period, for maximizing K. For this purpose, a 
weight is assigned for individual probabilities for election of $\mathrm{CHs}$ for advance and normal nodes; Fig.5. Therefore, SEP gives two different threshold formulae given in Eq.3 and Eq.5 [32-35]:

$\mathrm{T}\left(\mathrm{S}_{\text {nrm }}\right)=\left\{\begin{array}{cc}\frac{\mathrm{p}_{\text {nrm }}}{1-\mathrm{p}_{\text {nrm }}\left(\operatorname{rmod} \frac{1}{\mathrm{p}_{\text {nrm }}}\right)} & \text { ifS }_{\text {nrm }} \in \mathrm{G}^{\prime} \\ 0 & \text { otherwise }\end{array}\right.$

Where $\mathrm{G}^{\prime}$ is the set of normal nodes which can become $\mathrm{CH}$ and

$$
\begin{aligned}
& \mathrm{p}_{\mathrm{nrm}}=\frac{\mathrm{P}_{\text {opt }}}{1+\mathrm{a} \cdot \mathrm{m}} \\
& \mathrm{T}\left(\mathrm{S}_{\text {adv }}\right)=\left\{\begin{array}{cc}
\frac{\mathrm{p}_{\text {adv }}}{1-\mathrm{p}_{\text {adv }}\left(\operatorname{rmod} \frac{1}{\mathrm{p}_{\text {adv }}}\right)} & \text { ifS }_{\text {adv }} \in \mathrm{G}^{\prime \prime} \\
0 & \text { otherwise }
\end{array}\right.
\end{aligned}
$$

Where $\mathrm{G}^{\prime \prime}$ is set of advance nodes, which can become $\mathrm{CH}$ and

$$
\mathrm{p}_{\mathrm{adv}}=\frac{\mathrm{P}_{\mathrm{opt}}}{1+\mathrm{a} \cdot \mathrm{m}}(1+\alpha)
$$

The main limitations of SEP are [5]: Election of the cluster heads from the two type of nodes is not dynamic, due to which the nodes which are far from the strong nodes will get die first.

\subsection{DEEC Protocol}

DEEC [32-35] is another enhancement of LEACH for multilevel heterogeneous environment with respect to level of energies in WSNs. In SEP, energy distribution for two levels, advance nodes and normal nodes, whereas, DEEC introduces multi-level heterogeneity for maximizing $\mathrm{K}$. The nodes having greater residual energy have more right to become a $\mathrm{CH}$. Therefore, $\mathrm{CH}$ formation in DEEC is based on residual energy of entire network and residual energy of the node that wants to become a $\mathrm{CH}$. SEP calculates optimum number of $\mathrm{CHs}$ from Eq. 4 and 6 for advance and normal nodes, respectively. While, in DEEC, for multilevel heterogeneous node energy environment, nodes with higher residual energy attains more chances to become a $\mathrm{CH}$, Fig.6. Therefore, DEEC calculate optimum number of $\mathrm{CHs}$ for each round from the two equations:

$P_{i}=\left\{\begin{array}{cc}\frac{P_{o p t} E_{i}(r)}{(1+a . m) \bar{E}(r)} & \text { if } S_{i} \text { is normal node } \\ \frac{P_{\text {opt }}(1+a) E_{i}(r)}{(1+a . m)} \bar{E}(r) & \text { if } S_{i} \text { is advanced node }\end{array}\right.$

Where $\bar{E}(r)$ is the average energy of the network at round $r$ and is given by:

$\overline{\mathrm{E}}(\mathrm{r})=\frac{1}{\mathrm{~N}} \sum_{\mathrm{i}=1}^{\mathrm{N}} \mathrm{E}_{\mathrm{i}}(\mathrm{r})$

$E_{i}(r)$ Is the residual energy of the node at round $r$. Based on $\mathrm{Pi}$, DEEC calculates threshold as:

$\mathrm{P}\left(\mathrm{S}_{\mathrm{adv}}\right)=\frac{\mathrm{P}_{\mathrm{opt}} \mathrm{N}\left(1+\alpha_{\mathrm{i}}\right)}{\left(\mathrm{N}+\sum_{\mathrm{i}=1}^{\mathrm{N}} \alpha_{\mathrm{i}}\right)}$

DEEC evaluates that if the residual energy of the node is greater than the average energy of the network, then it has more chances to become a $\mathrm{CH}$. Thus, energy is well distributed in the network as it evolves. The main limitations of DEEC are: Advanced nodes always penalize in the DEEC, particularly when their residual energy reduced and become in the range of the normal nodes. In this position, the advanced nodes die rapidly than the others.

\subsection{For improved LEACH, iSEP, iTEEN and iDEEC algorithms:}

Rasheed et al. [34] proposed Energy-efficient whole Removing Mechanism (E-HORM) technique to remove energy holes. In this technique, they used sleep and awake mechanism for sensor nodes to save energy. This approach finds the maximum distance nodes to calculate the maximum energy for data transmission. They consider it as a threshold energy Eth. Every node first checks its energy level for data transmission. If the energy level of node is less than Eth, it cannot transmit data.

\subsection{1 for DDEEC Algorithm}

DDEEC [36] is based on DEEC scheme, where all nodes use the initial and residual energy level to define the cluster heads. To evade that each node needs to have the global knowledge of the networks, DDEEC like DEEC estimate the ideal value of network lifetime, which is used to compute the reference energy that each node should expend during each round. In the scheme, the network is organized into a clustering hierarchy, and the cluster heads collect measurements information from cluster nodes and transmit the aggregated data to the base station directly. Moreover, the authors have supposed that the network topology is fixed and no-varying on time, Fig.7. The difference between DDEEC and DEEC is localized in the expression which define the probability to be a cluster head for normal and advanced nodes. Simulation results show that the protocol performs better than the SEP and DEEC in terms of network lifetime and first node dies.

\section{PROPOSED ALGORITHM}

In this section, we present the details of proposed protocol. Our protocol implements the idea of probabilities for cluster heads selection based on initial energy and residual energy of sensor nodes as well as the average energy of the sensor network. Proposed algorithm consider three types of nodes every type have different initial energy level. Normal nodes have $E_{0}$ energy $m$ advanced nodes have a times energy more than normal nodes with $\mathrm{E}_{0}(1+\mathrm{a})$ energy level. $\mathrm{m}_{0}$ Super nodes have $b$ times energy more than normal nodes with $\mathrm{E}_{0}(1+\mathrm{b})$ energy level, where " $\mathrm{a}$ " and " $\mathrm{b}$ " are energy factors. As $\mathrm{N}$ is the number of total nodes in network, then for number of normal nodes, advanced nodes and super nodes $\mathrm{N}(1-\mathrm{m})$ , $\mathrm{Nm}\left(1-\mathrm{m}_{0}\right)$ and $\mathrm{Nmm}_{0}$ in the network, respectively.The total initial energy for normal, advanced and super nodes calculated as:

$$
\begin{aligned}
& E_{\text {normal }}=N(1-m) E_{0} \\
& E_{\text {advanced }}=N m\left(1-m_{0}\right) E_{0}(1+a) \\
& E_{\text {super }}=\mathrm{Nmm}_{0} E_{0}(1+b)
\end{aligned}
$$

The total initial energy for three level heterogeneity network calculated as:

$$
\begin{aligned}
& \mathrm{E}_{\text {total }}=\mathrm{E}_{\text {normal }}+\mathrm{E}_{\text {advanced }}+\mathrm{E}_{\text {super }} \\
& \mathrm{E}_{\text {total }}=\mathrm{N}(1-\mathrm{m}) \mathrm{E}_{0}+\mathrm{Nm}\left(1-\mathrm{m}_{0}\right) \mathrm{E}_{0}(1+\mathrm{a})+ \\
& \mathrm{Nmm}_{0} \mathrm{E}_{0}(1+\mathrm{b})
\end{aligned}
$$

A homogenous wireless sensor network after some rounds turns into heterogeneous due to the residual energy for nodes in network become different as compared to each other. Therefore, a protocol treat heterogeneity more important than homogenous protocol. Our proposed algorithm implements 
the same method for estimating the energy in the network as in DEEC. Since the probabilities calculated depend on the average energy at round $r$ in the network. This average energy estimated as:

$\overline{\mathrm{E}}(\mathrm{r})=\frac{1}{\mathrm{~N}} \mathrm{E}_{\text {total }}\left(1-\frac{\mathrm{r}}{\mathrm{R}}\right)$

Total rounds for network life time calculated as:

$\mathrm{R}=\frac{\mathrm{E}_{\text {total }}}{\mathrm{E}_{\text {round }}}$

Where $E_{\text {round }}$ energy dissipated during a single round in a network and calculated as:

$\mathrm{E}_{\text {round }}=\mathrm{L}\left(2 \mathrm{NE}_{\text {elec }}+\mathrm{NE}_{\mathrm{DA}}+\mathrm{kE}_{\mathrm{amp}} \mathrm{d}_{\mathrm{toBS}}^{4}+\mathrm{NE}_{\mathrm{fs}} \mathrm{d}_{\mathrm{toCH}}^{2}\right)$

where $\mathrm{k}$ is number of clusters, $\mathrm{d}_{\mathrm{toBS}}$ average distance between $\mathrm{CH}$ and $\mathrm{BS}, \mathrm{d}_{\mathrm{toCH}}$ is average distance between cluster head and cluster member. Now:

$\mathrm{d}_{\mathrm{toCH}}=\frac{\mathrm{M}}{\sqrt{2 \pi \mathrm{k}}}, \mathrm{d}_{\mathrm{toBS}}=0.765 \frac{\mathrm{M}}{2}$

Calculating the derivative of $\mathrm{E}_{\text {round }}$ with respect to $\mathrm{k}$ to zero we get optimal number of clusters as:

$\mathrm{k}_{\mathrm{opt}}=\sqrt{\frac{\mathrm{N}}{2 \pi}} \frac{\mathrm{M}}{\mathrm{d}_{\mathrm{toB} \mathrm{S}}^{2}} \sqrt{\frac{\mathrm{E}_{\mathrm{fs}}}{\mathrm{E}_{\mathrm{amp}}}}$

Now we can detect the energy dissipated per single round by substituting Eq.16 and Eq.17 in Eq.15.Every round at the start, nodes decided to be cluster head or not depend on threshold value. This threshold value calculated as:

$\mathrm{T}\left(\mathrm{S}_{\mathrm{i}}\right)=\left\{\begin{array}{cl}\frac{\mathrm{P}_{\mathrm{i}}}{1-\mathrm{P}_{\mathrm{i}}\left(\bmod \left(\mathrm{r}, \frac{1}{\mathrm{P}_{\mathrm{i}}}\right)\right)} & \text { ifS }, \mathrm{G} \\ 0 & \text { otherwise }\end{array}\right.$

Where $\mathrm{Pi}$ is the desired percentage of cluster heads, $\mathrm{r}$ is the current round number and $G$ is set of nodes haven't been cluster head in the last 1/prounds.In heterogeneity each type of nodes (normal, advanced and super nodes) have different probability. This probability calculated as:

$P_{i}=\left\{\begin{array}{lc}\frac{P_{\text {opt }} E_{i}(r)}{\left(1+m \cdot\left(a+m_{0} \cdot b\right)\right) \bar{E}(r)} & \text { ifS } S_{i} \text { isnormalnode } \\ \frac{P_{\text {opt }}(1+a) E_{i}(r)}{\left(1+m \cdot\left(a+m_{0} \cdot b\right)\right) \bar{E}(r)} & \text { ifS }{ }_{i} \text { isadvancednode } \\ \frac{P_{\text {opt }}(1+b) E_{i}(r)}{\left(1+m \cdot\left(a+m_{0} \cdot b\right)\right) \bar{E}(r)} & \text { ifS }{ }_{i} \text { issupernode }\end{array}\right.$

Threshold for $\mathrm{CH}$ selection is calculated for each type as:

$\mathrm{T}\left(\mathrm{S}_{\mathrm{i}}\right)=\left\{\begin{array}{cc}\frac{\mathrm{P}_{\mathrm{i}}}{1-\mathrm{P}_{\mathrm{i}}\left(\bmod \left(\mathrm{r}, \frac{1}{\mathrm{P}_{\mathrm{i}}}\right)\right)} & \text { if } \mathrm{S}_{\mathrm{i}} \in \mathrm{G} \\ \frac{\mathrm{P}_{\mathrm{i}}}{1-\mathrm{P}_{\mathrm{i}}\left(\bmod \left(\mathrm{r}, \frac{1}{\mathrm{P}_{\mathrm{i}}}\right)\right)} & \text { if } \mathrm{S}_{\mathrm{i}} \in \mathrm{G}^{\prime \prime} \\ \frac{\mathrm{P}_{\mathrm{i}}}{1-\mathrm{P}_{\mathrm{i}}\left(\bmod \left(\mathrm{r}, \frac{1}{\mathrm{P}_{\mathrm{i}}}\right)\right)} & \text { if } \mathrm{S}_{\mathrm{i}} \in \mathrm{G}^{\prime \prime \prime} \\ 0 & \text { otherwise }\end{array}\right.$

G Is the set of normal nodes haven't become $\mathrm{CH}$ at last $1 / \mathrm{P}_{\mathrm{i}}$ rounds of the epoch where $S_{i}$ is normal node.G" Is the set of advanced nodes haven't become $\mathrm{CH}$ at last $1 / \mathrm{P}_{\mathrm{i}}$ rounds of the epoch where $S_{i}$ is advanced node. $G^{\prime \prime \prime}$ Is the set of super nodes haven't become $\mathrm{CH}$ at last $1 / \mathrm{P}_{\mathrm{i}}$ rounds of the epoch where $\mathrm{S}_{\mathrm{i}}$ is super node. $1 / \mathrm{P}_{\mathrm{i}}$. Thus, the probabilistic threshold value of cluster head depends on initial energy, residual energy and type of a sensor node. The $\mathrm{CHs}$ act as local control center to organize and manage the data transmission in their cluster.
CHs transmit TDMA schedule to the sensor nodes in their cluster. That make no collision during the transmission of data messages. $\mathrm{CHs}$ permit radio component of each non $\mathrm{CH}$ sensor node to be turnoff at all-time except among transmission time, thus minimize energy consumption for each sensor node in the network, Fig.8.

\section{RESULTS AND DISCUSSIONS}

The performance of all discussed algorithms will be tested and simulated using the following parameters of the WSNs: (i) Number of Sensor Nodes: 100 sensor nodes in a field of $100 \times 100 \mathrm{~m}$; (ii) Nodes are deployed random; (iii) base station is centered in the field and has unlimited power, and (iv) Ignore the effect caused by interference and signal collision in the wireless channel. IN this section we assume: $\mathrm{m}=0.5$, $\mathrm{m}_{0}=0.4, \mathrm{a}=1.5$, and $\mathrm{b}=3$ for this we consider a network contain 50 normal nodes, 30 advanced nodes and 20 super nodes. Advanced nodes having 1.5 times more energy than normal nodes, super nodes having 3 times more energy than normal nodes and other parameters shown in Table.1. Fig.9 shows the number of nodes alive and dead nodes during the network life time: (i) the first node for LEACH; ILEACH; SEP; ISEP; TEEN; ITEEN; DEEC; IDEEC; DDEEC, and the proposed algorithm dies at $385 ; 484 ; 467 ; 566 ; 829 ; 1125$; $1219 ; 1238 ; 1252$, and 1368 rounds, respectively, and (ii) all nodes die at $792 ; 1877 ; 1888 ; 2399 ; 2288 ; 3184 ; 3441 ; 3575$; 4999, and 9958. Fig.10 shows that the data sent to BS is more for the proposed algorithm as compared to all other protocols. It is clear our protocol is the most efficient from the results among the given protocols in terms of network lifetime, stability period and number of data packets sent to the BS. The proposed algorithm performs better than the existing protocols in terms of stability period, network lifetime and throughput. During protocols operations, LEACH and TEEN are homogenous routing protocols and all nodes in network have same probability to become cluster head. SEP assumes that in real environment sensor nodes having different energy level, therefore SEP use two types of sensor nodes normal nodes and advanced nodes. In DEEC protocol all nodes use residual and initial energy level to select the $\mathrm{CH}$, DEEC considers average energy of the network. DDEEC uses same method for calculation of average energy in the network and cluster head selection algorithm based on residual energy as presented in DEEC. Difference between DEEC and DDEEC in expression that define probability for advanced and normal nodes to be a $\mathrm{CH}$. The proposed algorithm consumes less energy which leads to prolong life time of the network and prolong stability period that means sensor nodes are able to send data packets for time, thereby the number of packets sent to Base Station are more comparison to the other selected protocols.

Table 1. Simulation Parameters

\begin{tabular}{|l|l|}
\hline Parameters & Value \\
\hline Network field & $100 \mathrm{~m} * 100 \mathrm{~m}$ \\
\hline Number of nodes & 100 \\
\hline Initial energy of normal nodes $\mathrm{E}_{0}$ & $0.5 \mathrm{j}$ \\
\hline Message size & $4000 \mathrm{Bit}$ \\
\hline $\begin{array}{l}\mathrm{E}_{\text {elec }}(\text { receiver energy per node(ERX) } \\
\text { transmitter energy per node }(\mathrm{ETX}))\end{array}$ & $50 \mathrm{~nJ} / \mathrm{bit}$ \\
\hline $\begin{array}{l}\mathrm{E}_{\mathrm{fs}}(\text { amplification energy when } \mathrm{d} \text { is less } \\
\left.\text { than } \mathrm{d}_{0}\right)\end{array}$ & $10 \mathrm{nj} / \mathrm{bit} / \mathrm{m}^{2}$ \\
\hline
\end{tabular}




\begin{tabular}{|l|l|}
\hline $\begin{array}{l}\mathrm{E}_{\text {amp }}(\text { amplification energy when } \mathrm{d} \text { is } \\
\left.\text { greater than } \mathrm{d}_{0}\right)\end{array}$ & $0.0013 \mathrm{pJ} / \mathrm{bit} / \mathrm{m}^{4}$ \\
\hline $\mathrm{E}_{\mathrm{DA}}$ (Data Aggregation Energy) & $5 \mathrm{~nJ} / \mathrm{bit} /$ signal \\
\hline $\mathrm{d}_{0}$ (Threshold Distance) & $70 \mathrm{~m}$ \\
\hline $\mathrm{P}_{\text {opt }}$ & 0.1 \\
\hline maximum number of rounds $(\mathrm{r})$ & 10000 \\
\hline
\end{tabular}

\section{CONCLUSIONS}

Designing an energy efficient routing protocol is a major challenge in WSNs. Data sending and reception was used to describe the energy utilization of the sensors. Hence, the protocols designed for WSNs should be as energy efficient as much as possible so that it will extend the lifetime of each sensor, and hence the lifetime of network. Each of the discussed routing protocol has its own advantages and disadvantages, based on the network topology the routing protocols can be applied. In this paper, we increased the level of WSNs heterogeneity, through the addition of super node, having energy more than advanced nodes; normal nodes, and respective probabilities. Residual energy and initial energy is the main concept to elect $\mathrm{CH}$ from sensor nodes. The performance of four traditional techniques (LEACH; SEP; TEEN, and DEEC); five modified techniques (ImprovedLEACH; Improved-SEP; Improved-TEEN; Improved-DEEC, and Developed-DEEC), as well as a proposed technique, was evaluated using four matrices: stability period; network lifetime; number of packets sent to BS, and throughput. As a result of the extensive experimental results, it was found that the proposed routing protocol is superior to all other discussed nine techniques.

\section{REFERENCES}

[1] K. Punjani and S.S. Tyagi, "Energy Efficient Routing Protocols For Wireless Sensor Network," International Journal of Engineering Research \& Technology (IJERT), Vol.2, Issue.5, pp: 1936-1942, May - 2013.

[2] R. Dureja and M. Malik, "Routing in Wireless Sensor Networks: A Review," International Journal of Emerging Research in Management \&Technology, Vol.4, Issue.0, pp:124-129, October - 2015.

[3] M. Abdullah and A. Ehsan, "Routing Protocols for WSNs: Classifications \& Challenges," Quest Journals, Journal of Electronics and Communication Engineering Research, Vol.2, Issue.2, pp: 5-15, 2014.

[4] M. Chaudhary, "Wireless Sensor Networks: A Comprehensive Comparison of Routing Protocols and Energy Efficient Techniques," Communications on Applied Electronics (CAE), Foundation of Computer Science FCS, Vol.2, No.1, pp: 1-9, June - 2015.

[5] Dheeraj, and Ritu Mishra, "Review Paper on Hierarchal Energy- Efficient Protocols in Wireless Sensor Networks," International Journal of Advanced Research in Computer Science and Software Engineering, Vol.4, Issue.6, pp: 262-266, 2014, ISSN: 2277-128X.

[6] P. Thakur, "Clustering Schemes in Wireless Sensor Networks and Mobile Adhoc Network: Classification and Comparison," IRACST - International Journal of Computer Networks and Wireless Communications (IJCNWC), Vol.2, No.6, pp: 671-675, 2012.

[7] S. Sharma and M.Rani, "A Survey and Comparative
Study of Routing Protocols in Wireless Sensor Network," Int. Journal of Engineering and Computer Science, Vol.3, Issue.5, and pp: 6008-6012, 2014.

[8] N. Saini and J. Singh, "A Survey: Hierarchal Routing Protocol in WSNs," Global Journal of Computer Science \& Technology, Vol.14, Issue.1, pp: 33-39, 2014

[9] P.K. Poonguzhali, N.P. Ananthamoorthy, S.J. Jabakani, "Design Challenges and Comprehensive Study on Cluster Based Routing Protocol in Wireless Sensor Network," Int. Journal of Advanced Research in Computer and Communication Engineering, Vol.4, Issue.1, pp: 5-11June 2015.

[10] P. Kumarawadu, D.J. Dechene, M. Luccini, A. Sauer, "Algorithms for Node Clustering in Wireless Sensor Networks: A Survey. In Proceedings of 4th International Conference on Information and Automation for Sustainability, Colombo, Sri Lanka, 12-14 December 2008; pp: 295-300.

[11] I. Panda, "QoS Parameters Analysis to Improve QoS in WSNs Routing Protocol," Int. Journal of Advanced Research in Computer Engineering \& Technology, Vol.1, Issue.8, and pp: 105-114, 2012.

[12] B.P. Deosarkar, N.S. Yada, R.P. Yadav, "Cluster Head Selection in Clustering Algorithms for Wireless Sensor Networks: A Survey," In Proceedings of the 2008 International Conference on Computing, Communication and Networking, Virgin Islands, USA, 3-7 August 2008; pp: $1-8$.

[13] C. Jiang, D. Yuan, Y. Zhao, "Towards Clustering Algorithms in Wireless Sensor Networks - A Survey," In Proceedings of IEEE Wireless Communications and Networking Conference, Budapest, Hungary, 5-8 April 2009; pp: 1-6.

[14] M. Maimour, H. Zeghilet and F. Lepage, Cluster-based Routing Protocols for EnergyEfficiency in Wireless Sensor Networks, Sustainable Wireless Sensor Networks, Yen Kheng Tan (Ed.), ISBN: 978-953-307297-5, 2010.

[15] S. Misra et al. (eds.), Guide to Wireless Sensor Networks, Computer Communications and Networks, DOI: $\quad 10.1007 / 978-1-84882-218-4 \quad 4$, Springer-Verlag London Limited 2009.

[16] S.K. Singh, M.P. Singh, and D.K. Singh,"Routing Protocols in Wireless Sensor Networks -A Survey," Int. Journal of Computer Science \& Engineering Survey, Vol.1, No.2, and pp: 63-83, 2010.

[17] P. Ramesh and S. Kruba," A Comparative Study on Various Routing Protocols of Wireless Sensor Network," Proceedings of the UGC Sponsored National Conference on Advanced Networking and Applications, PP: 202205, 27 March 2015.

[18] Brijbhushan and S. Anand," A Survey- Wireless Sensor Networks Routing Protocols,'International Journal of Advanced Research in Electronics and Communication Engineering, Vol.4, Issue.2, and pp: 152-158, 2015.

[19] R. Sujeeand K.E. Kannammal,"Routing protocols based on network structure in wireless sensor networks -A survey," SSRG Int. Journal of Computer Science and Engineering, Vol.2, Issue.4, pp: 38-45, April 2015. 
[20] P. Krishnaveni and J. Sutha, "Analysis Of Routing Protocols For Wireless Sensor Networks," Int. Journal of Emerging Technology and Advanced Engineering, Vol.2, Issue.11, and pp: 401-407, 2012.

[21] T. Sharma, H. Singh, and A. Sharma, "A Comparative Review on Routing Protocols in Wireless Sensor Networks," Int. Journal of Computer Applications, Vol.123, No.14, and pp: 28-33, 2015.

[22] Mr.Sushil R. Sawdekar and Prof. C.M.Mankar, "A Review of Routing Protocols for Wireless Sensor Network," International Journal of Engineering Sciences \& Research Technology, Vol.3, No.1, pp: 424-429, 2014.

[23] J. Zheng and A. Jamalipour, "Wireless Sensor Networks: A Networking Perspective," a book published by A John \& Sons, Inc, and IEEEE, 2009.

[24] J. Al-Karaki, and A.E. Kamal, "Routing Techniques in Wireless Sensor Networks: Asurvey, "IEEE Communications Magazine, Vol.11, No.6, pp: 6-28, 2004.

[25] K. Akkaya and M. Younis, "A Survey on Routing Protocols for Wireless Sensor Networks," Ad hoc Networks, Vol.3, No.3, and pp: 325-349, 2005.

[26] J.N. Al-Karakin and A.E. Kamal, "Routing Techniques in Wireless Sensor Networks: A Survey," IEEE Wireless Communication, Vol.11, pp: 6-28, 2004.

[27] N. Kaur, D. Sharma, and P. Singh, "Classification of Hierarchical Routing Protocols in Wireless Sensor Network: A Survey," International Journal of P2P Network Trends and Technology (IJPTT), Vol.3, Issue.1, pp: 56-61, 2012.

[28] O. Rehman, N. Javaid, B. Manzoor, A. Hafeez, A. Iqbal, and M. Ishfaq, "Energy Consumption Rate basedStable Election Protocol (ECRSEP) for WSNs," International Workshop on Body Area Sensor Networks in conjunction with 4th International Conference on Ambient Systems, Networks and Technologies, 2013, Halifax, Nova Scotia, Canada,PP: 932 - 937 1877-0509 (c) 2013 .

[29] O.Deepa and A. Senthilkumar,"Classification of
Hierarchical Routing Protocols in Wireless Sensor Network," International Conference on Interdisciplinary Research in Electronics and Instrumentation Engineering [ICIREIE 2015], volume (1), pp: 84-89.

[30] C. Rajivegandhi, K. Raghavi, and K.S. Jeyshree,” Low Energy Adaptive Clustering Hierarchy based routing Protocols Comparison for Wireless Sensor Networks," IOSR Journal of Computer Engineering (IOSR-JCE), Vol.16, Issue.6, Ver(2), pp: 56-61, 2014.

[31] G. Chandini, R. Guntur., and K.W. Guntur, "Energy Efficient Zonal Stable Election Protocol for WSNs," International Journal of Advanced Research in Electronics and Communication Engineering (IJARECE) Vol.3, Issue.12, and pp: 1900-1905, 2014.

[32] K.Latif, M.Jaffar, N.Javaid, M.N.Saqib, U.Qasim, Z.A.Khan, "Performance Analysis of Hierarchical Routing Protocols in Wireless Sensor Networks," NGWMN with 7th IEEE Int. Conf. on Broadband and Wireless Computing, Communication and Applications, Canada, 2012.

[33] N. Amjad, M.M. Sandhu, et al., "DREEM-ME: Distributed Regional Energy Efficient Multi-hop Routing Protocol based on Maximum Energy with Mobile Sink in WSNs," Journal of Basic and Applied Scientific Research Vol.4, Issue.1, pp: 289-306, 2014.

[34] M.B. Rasheed, N. Javaid, Z.A. Khan, U. Qasim, and M. Ishfaq, "E-Horm: An Energy-Efficient Hole Removing Mechanism Inwireless Sensor Networks," Electrical and Computer Engineering 26th Annual IEEE Canadian Conference, pp:1-4, 2013.

[35] A. Sikandar and S. Kumar, "Energy Efficient Clustering in Heterogeneous WSNs using Degree of Connectivity," International Journal of Computer Networks \& Communications, Vol.7, No.2, pp: 19-31,March - 2015.

[36] A. Rathee, I. Kashyap, and K. Choudhary,"Performance Evaluation of PSO based optimization of Distributed Energy-Efficient Clustering (DDEEC) algorithm in heterogeneous WSN," Vol.3, Issue.4, and pp: 248-256, April-2014.

\section{APPENDIX}

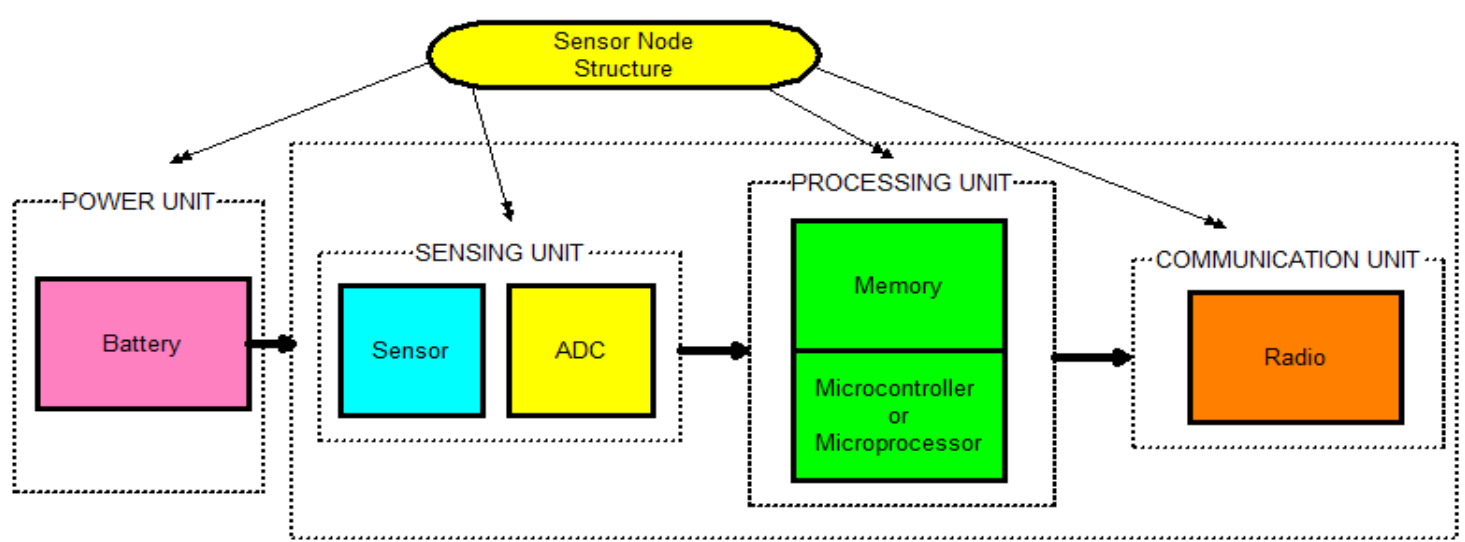

Fig 1: Sensor nodes structure 


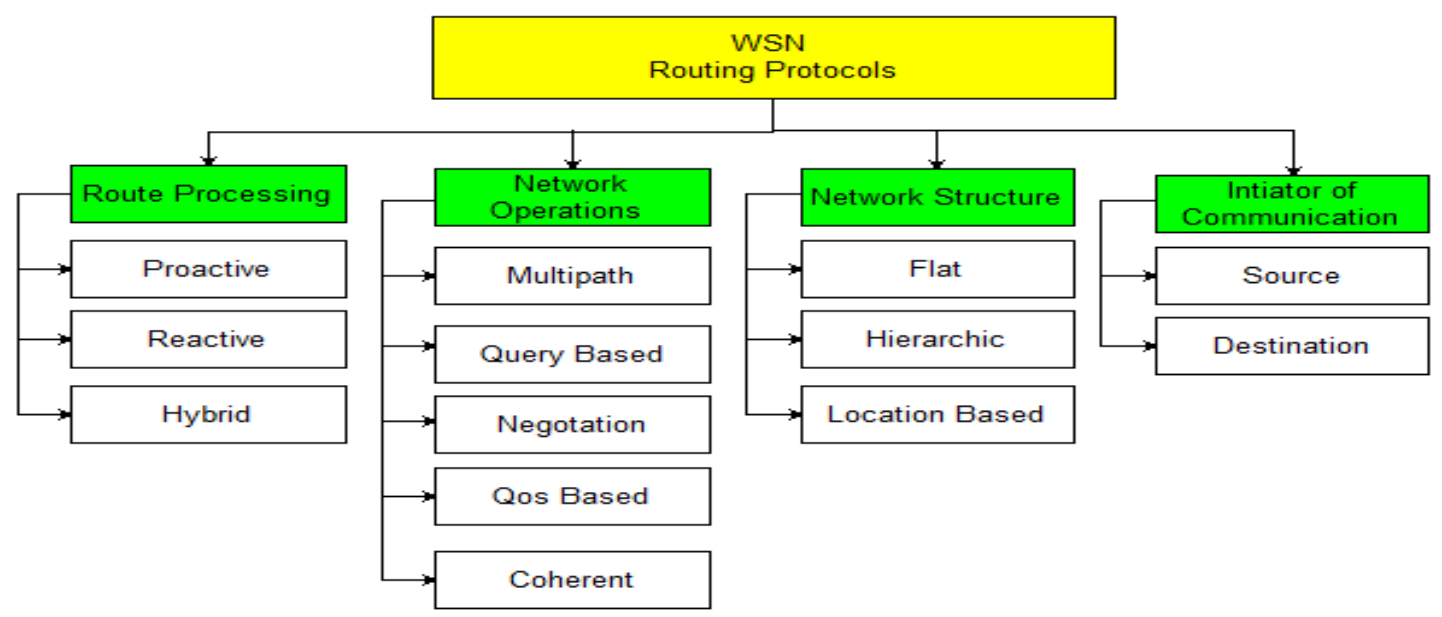

Fig 2: WSN routing protocols

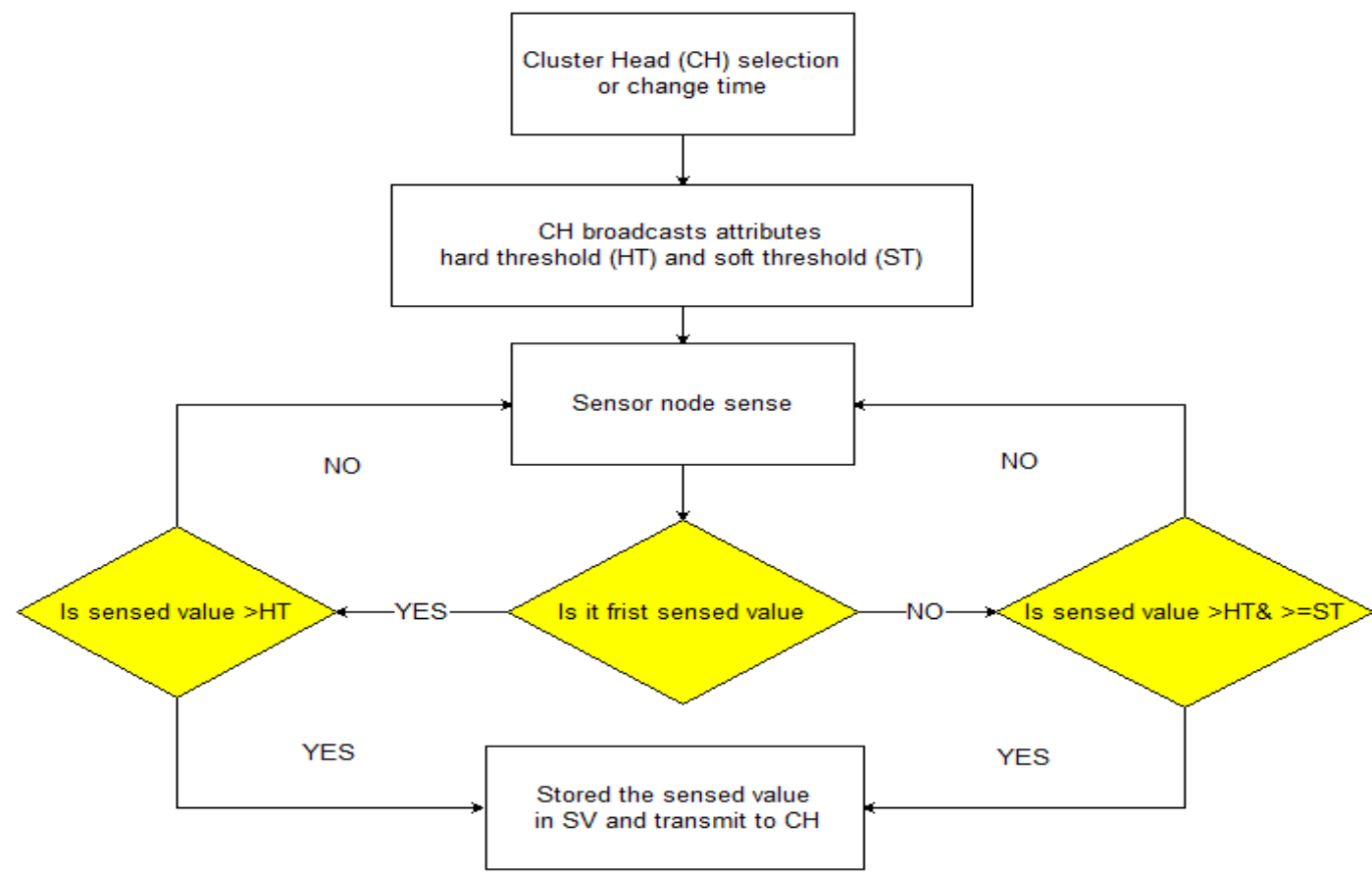

Fig 3: Flow chart for TEEN 


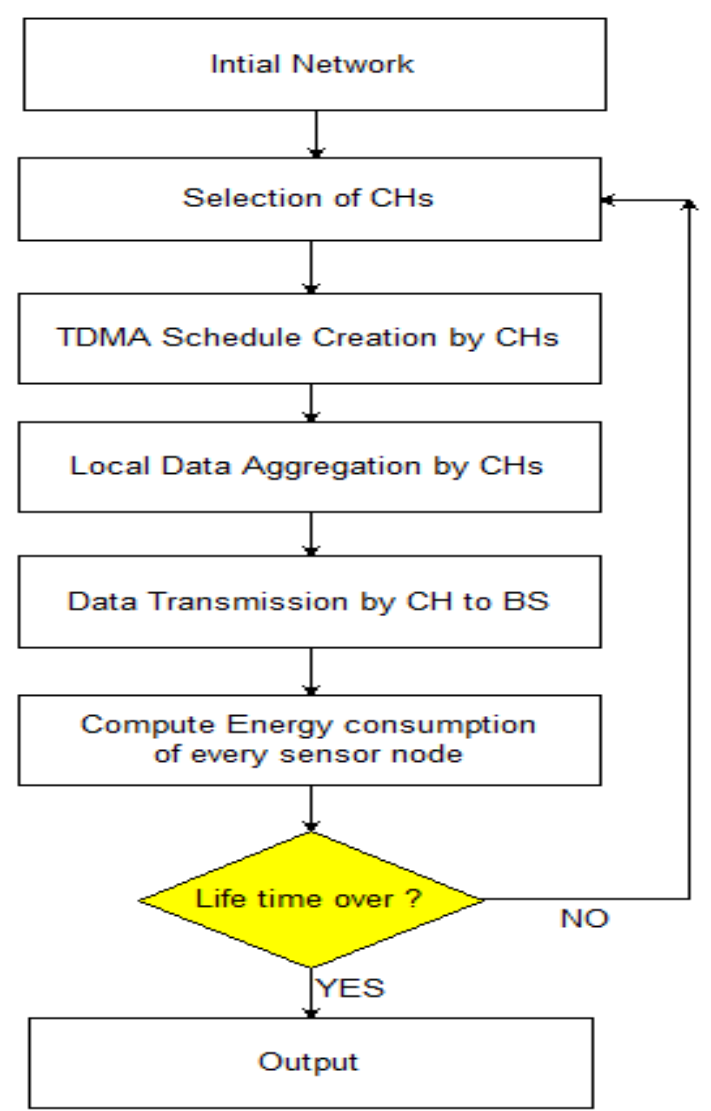

Fig 4: Flow chart for LEACH

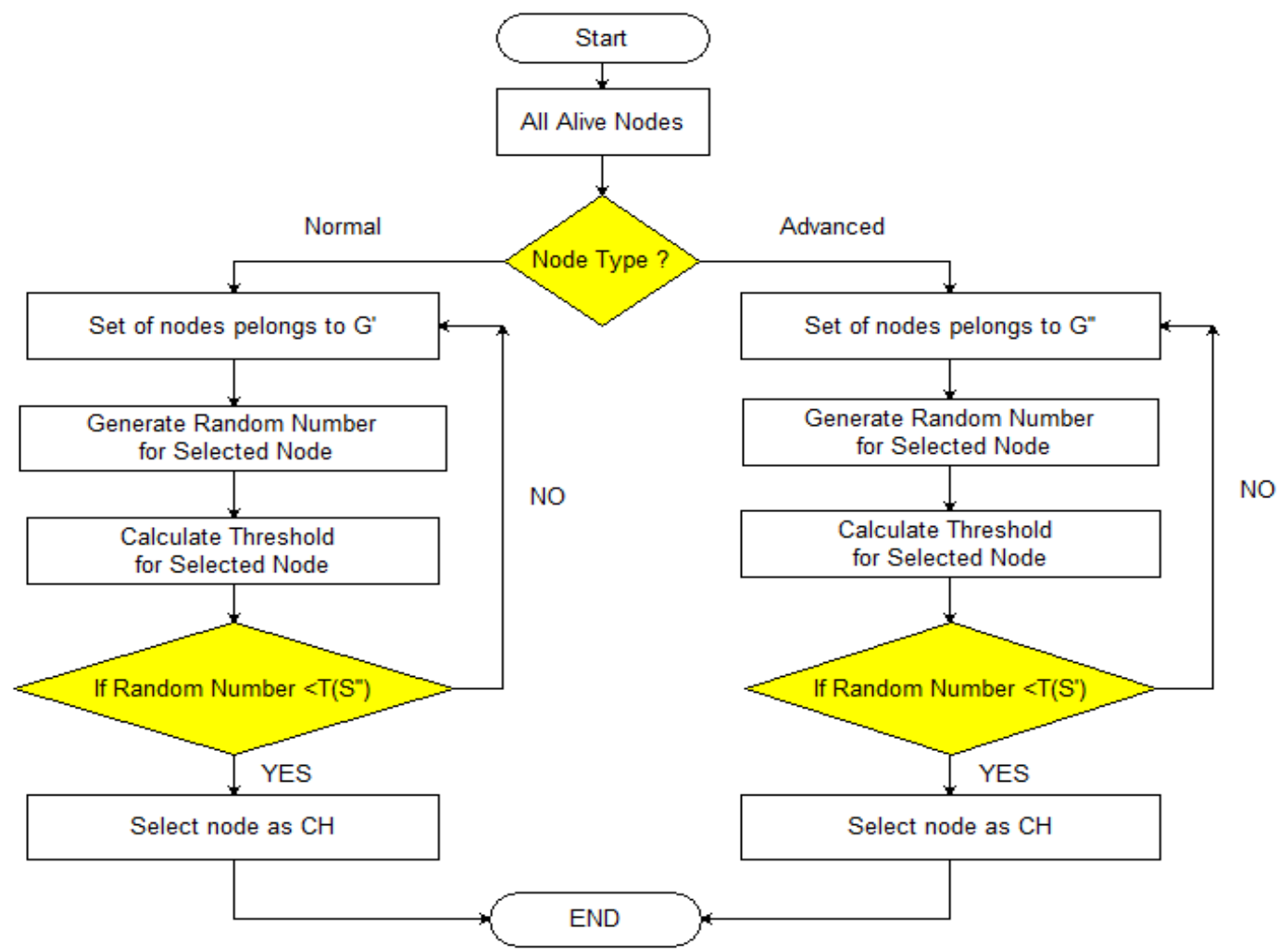

Fig 5: Flow chart for SEP 


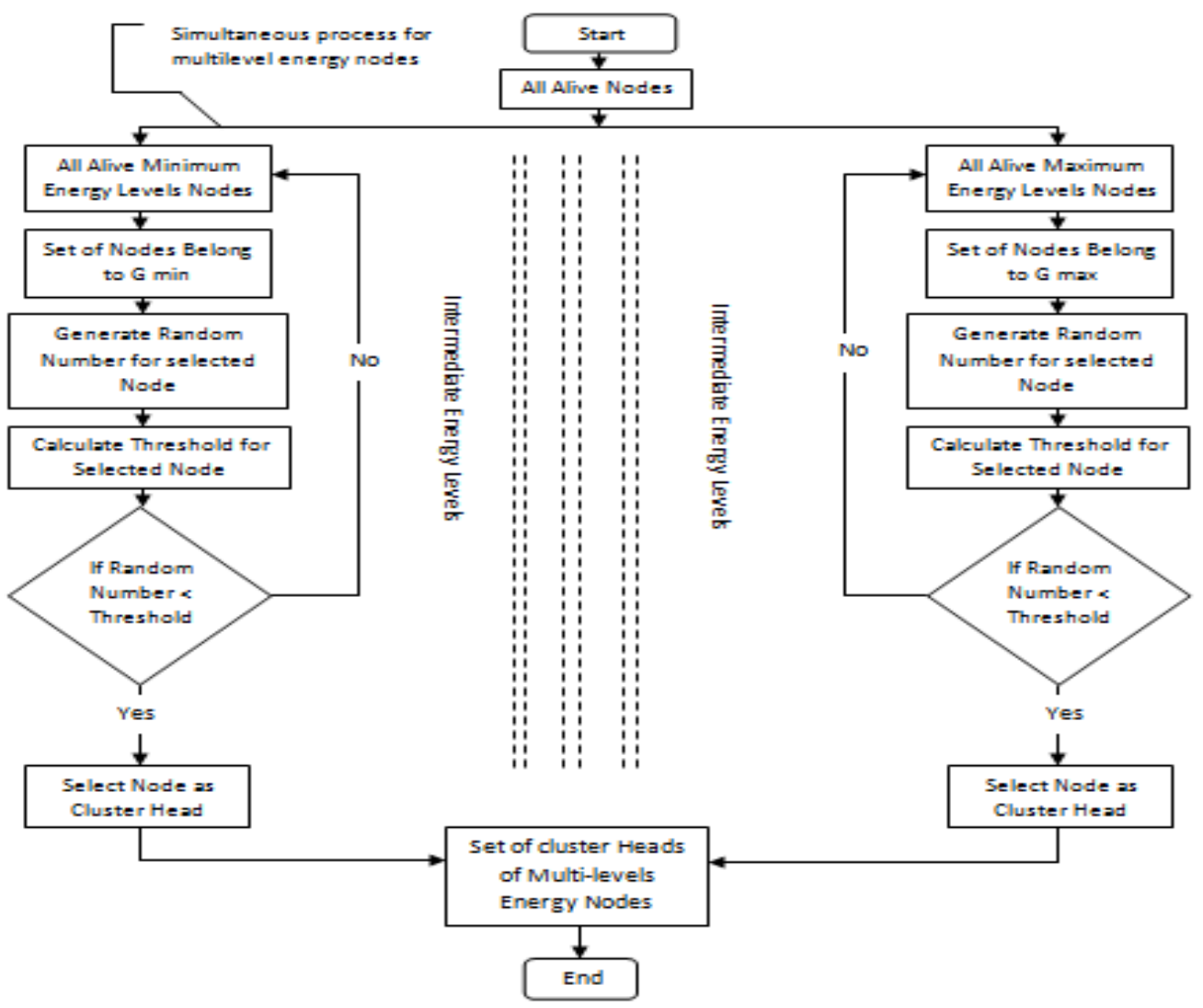

Fig 6: Flow chart for DEEC

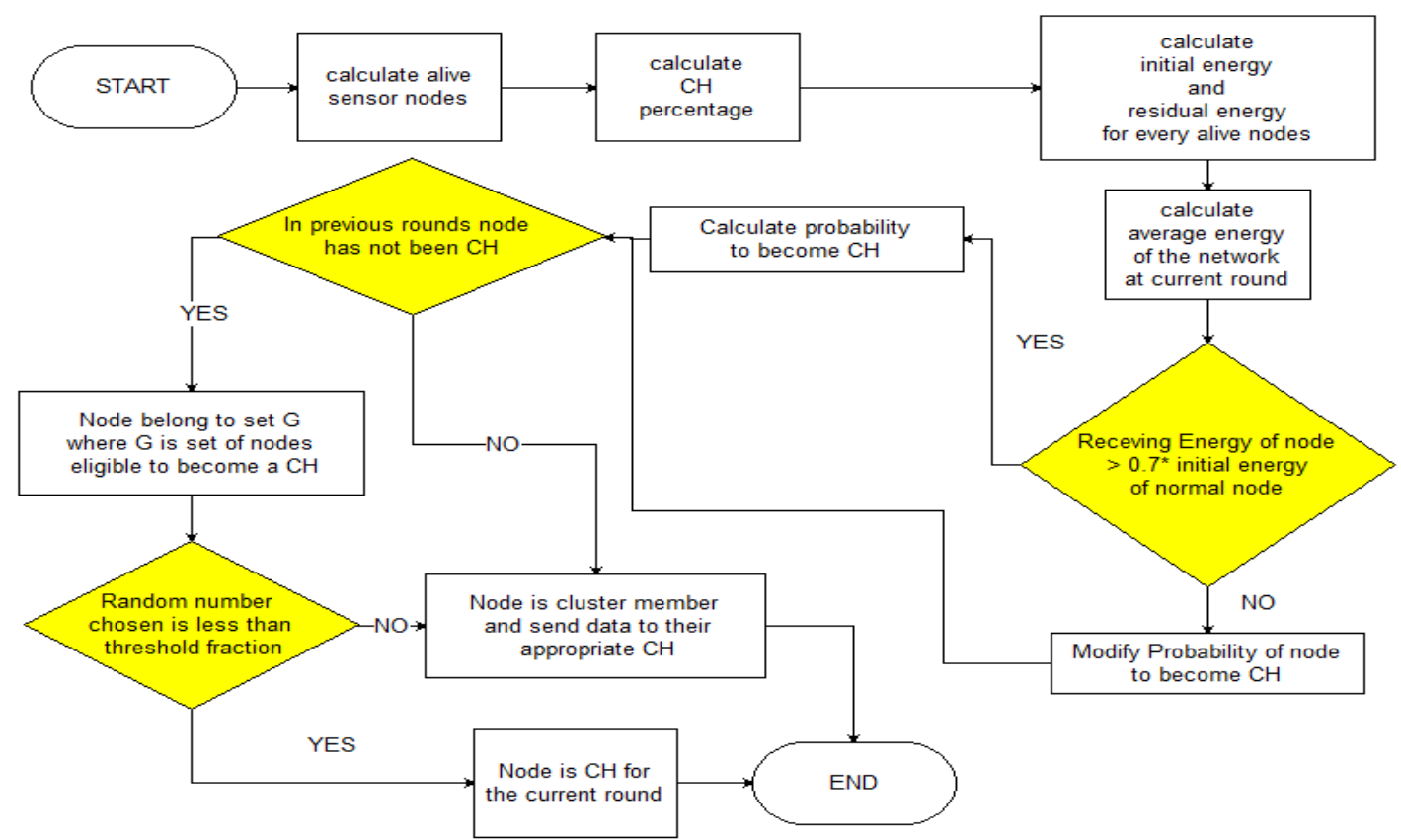

Fig 7: Flow chart for DDEEC 


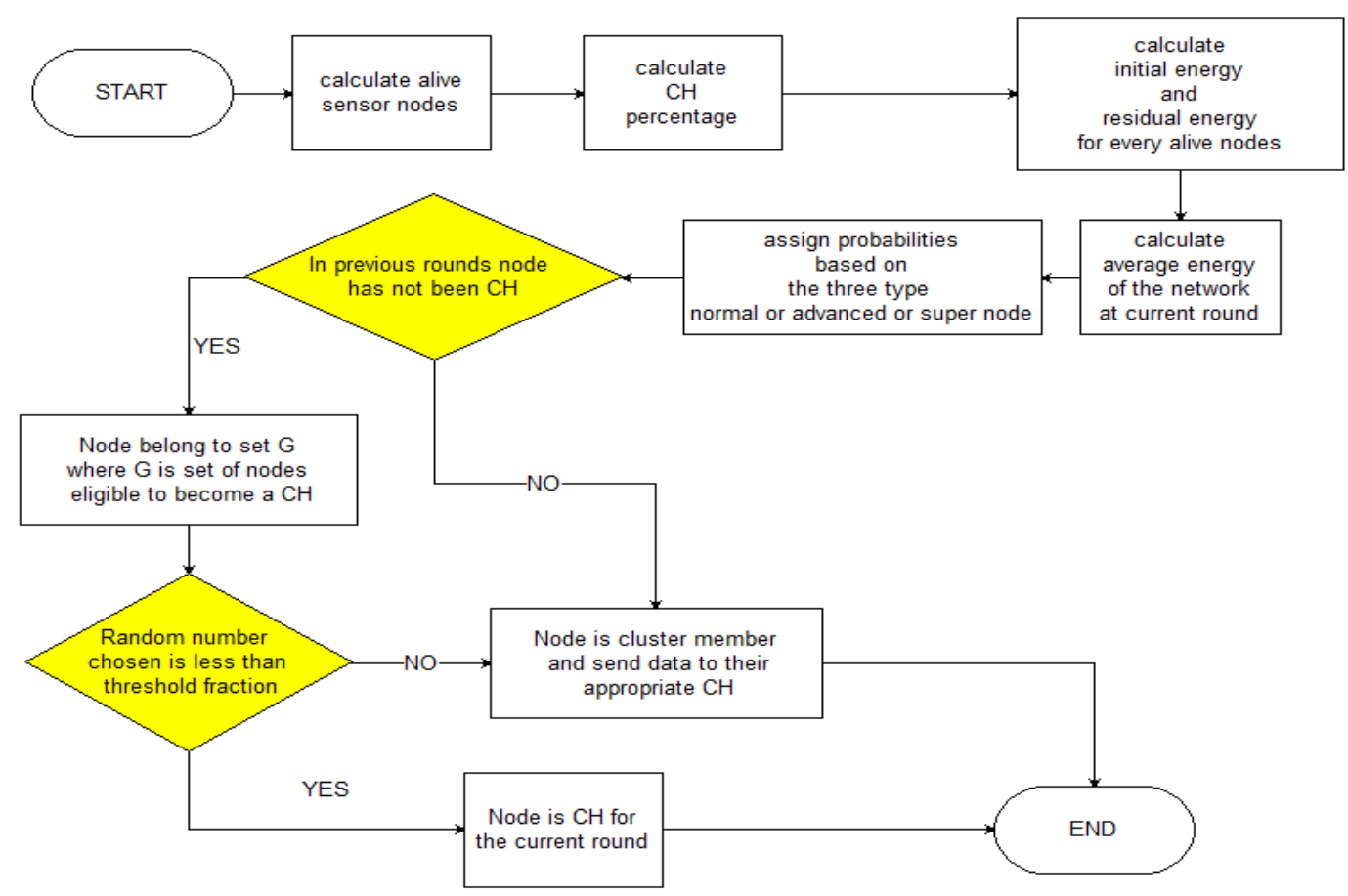

Fig 8: Flow chart for proposed algorithm

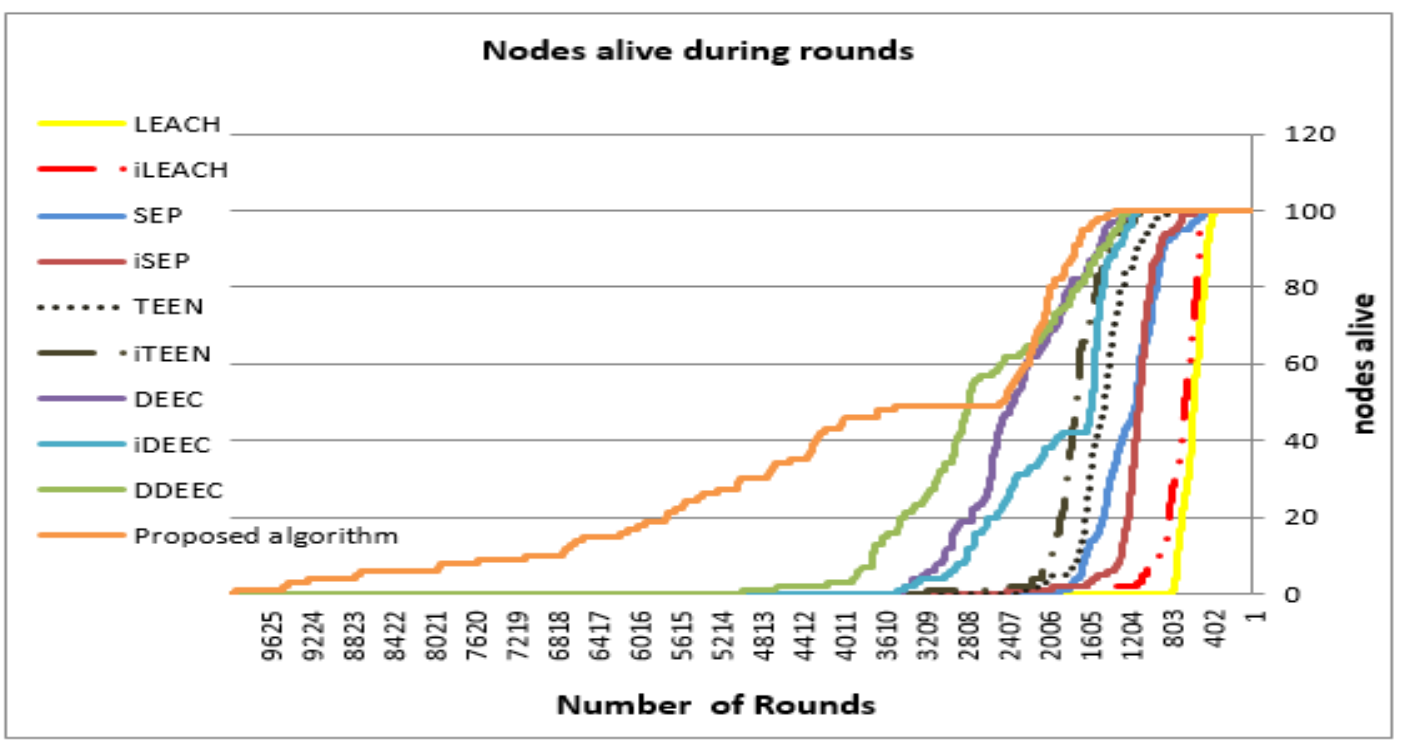

Fig 9: Results of nodes alive vs. round for all algorithms 


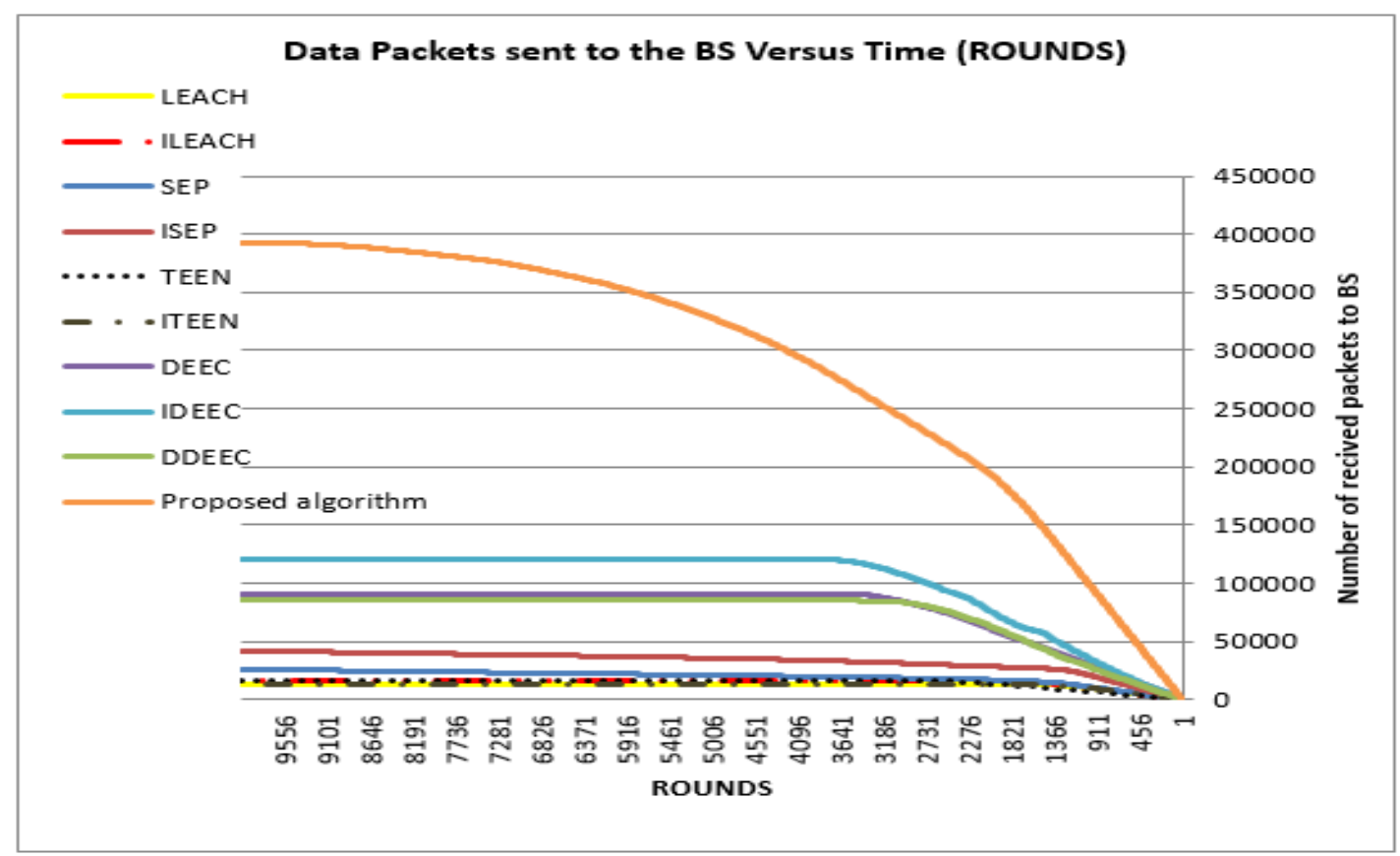

Fig 10: Data packets vs. rounds for all presented algorithms 